\title{
Nanotechnology approaches to self-organized bio-molecular devices
}

\author{
Roberto Cingolani *, Ross Rinaldi, Giuseppe Maruccio, Adriana Biasco \\ National Nanotechnology Laboratory of INFM (www.nnl.it) University of Lecce, Via Arnesano, 73100 Lecce, Italy
}

\begin{abstract}
In this paper we briefly describe new strategies to exploit self-assembled solid-state biomolecular materials as active elements of electronic devices. Two basically different approaches are proposed: a top-down approach, where biomolecular semiconductors consisting of DNA basis are self-organized and interconnected by planar metallic nanopatterns, and a bottom-up approach, where single or ordered matalloproteins are immobilized in a nanocircuit realizing a hybrid covalently bound biologic-inorganic system. The transport characteristics of different devices such as diodes, photodetectors and metalsemiconductor-metal structures will be described. (C) 2002 Elsevier Science B.V. All rights reserved.
\end{abstract}

Keywords: Molecular electronics; Nanotechnologies

Biomolecular electronics (BME) is raising increasing interest worldwide, due to the appealing possibility of realizing cheap and easy-to-fabricate devices exploiting the natural self-assembling, self-recognition and self-repairing capability of biological matter. Although very recent, BME has deep roots in the field of organic molecular electronics, whose flagships are carbon nanotubes and molecular junctions [1-4]. Biomolecules are in general more robust than other organic molecules, thus envisaging a more reliable utilization in electronic devices. Moreover, they are characterized by a number of unique electron transport phenomena, such as charge transfer in proteins, hopping and/or band-like transport $(\pi-\pi)$ in self-assembled systems. Finally, both their electronic structure and their ligands can be engineered in a very flexible way, thus allowing a fine tuning of the

\footnotetext{
* Corresponding author.

E-mail address: roberto.cingolani@unile.it (R. Cingolani).
}

HOMO-LUMO gap, of the oxidation potential, and of the selective bonding to different surfaces. Very recently, the attention of BME has been directed toward the identification of molecules combining good conductivity with good self-assembling and self-recognition properties. DNA has been one of the most investigated class of biomolecules [5-7], leading to a somewhat controversial description of its electrical properties, and, hence, of its potentiality for electronic applications. Depending on the interconnection mechanism (chemical bonding of the DNA on a metal by a selected sequence of oligonucleotides [5], mechanical contact with a gold interdigitated patterns [6] or single DNA molecule immobilized in a metal contact [7]) the DNA molecules have been found to be conductive, non-conductive or rectifying. From a totally different point of view, some groups are trying to use biological methods to control the formation of semiconductors and metals $[8,9]$ by investigating the peptide-driven formation of gold crystals, as a prototype mechanism for the formation 
of natural solids like bones and teeth in the human body. Brown et al. [10] have screened a combinatory library of millions of peptides to select specific peptide sequences which can distinguish among different crystallographic planes of the most important semiconductors used in technology ( $\mathrm{GaAs}$ and $\mathrm{Si}$ ). The peptides could therefore be used to control the positioning and the assembly of materials at nanoscale, which has a tremendous impact on future electronic technologies. High-efficiency photodetectors based on a solid-state self-organized DNA basis, whose figure of merits become appealing even for solar cell applications, have been recently demonstrated by Rinaldi et al. [11]. Finally, the group at Cornell [12] (and other groups worldwide) are trying to reconstruct nerves and interconnect them to circuits in view of future prosthetic applications.

The most surprising thing is that most of these groups have discovered the enormous potentiality of biomolecular self-assembling even though they started from different backgrounds and with totally different targets (spanning from molecular electronics to the basic understanding of bones formation in the human body). This gives a clear flavor of how general is the cultural revolution we are experiencing. In this paper, we discuss a possible strategy for the implementation of electronic devices based on self-organized biomolecules. We would like to discuss two possible approaches, which combine the self-organization and the immobilization of biomolecules in the solid state and the nanotechnologies adopted in semiconductor physics.

The top-down approach, is based on the selforganization of solid-state films of a DNA basis called deoxiguanosine, which interconnect planar metallic nanocircuits.

The bottom-up approach relies on the covalent hybrid bonding of a metalloprotein (Azurin), onto a planar nanopattern. The metal atom provides electrons for the transport, so that in principle a single molecule device can be fabricated.

\section{The top-down approach}

A drop of constant volume $(2 \mu \mathrm{l})$ of a deoxyguanosine solution in $\mathrm{CHCl}_{3}$ at low concentration (between $10^{-1}$ and $10^{-4} \mathrm{M}$ ) is deposited by a Hamilton siring in the gap of two metallic contacts and gently dried in low vacuum (base pressure around $10^{-3}$ bar). Upon controlled evaporation the molecules are found to form self-assembled guanosine crystals (SAGC) spontaneously. The metallic electrodes (60 nm gap in Fig. 1b), were prepared by Electron Beam Lithography in a $\mathrm{Au} / \mathrm{Cr}(100 \mathrm{~nm} / 8 \mathrm{~nm})$ film deposited on $\mathrm{SiO}_{2}$ substrate (roughness below $0.2 \mathrm{~nm}$ ). The deoxiguanosine molecules form the ribbon-like structure depicted in Fig. 1a. The AFM analysis (Fig. 1c) shows that the ribbons form an ordered supramolecular structure, laying parallel to each other with a periodicity of $2.5 \mathrm{~nm}$ and a length up to $l<100 \mathrm{~nm}$ (see Fig. 1c). For longer distances, such ordering is lost, and the random orientation of the SAGCs on the plane gives rise to an amorphous polycrystalline material.

The SAGCs behave like semiconductors [11], with equivalent energy gap of about $3.5 \mathrm{eV}$ at room temperature and electron effective mass of the order of $2 m_{0}$ (very similar to $\mathrm{GaN}$ ). The typical photocurrent spectrum of the SAGCs is shown in Fig. 2, showing a clear absorption onset at the energy gap.

The SAGCs were deposited onto different planar devices with gaps $L=60,120$ and $800 \mathrm{~nm}$. The narrower gap was small enough to probe transport within an individual SAGC, as this normally extends over $<100 \mathrm{~nm}$. The $120 \mathrm{~nm}$ gap was used to probe just a few (two or three) SAGCs, whereas the widest gap was used to probe an amorphous ensemble of SAGCs. All the current-voltage $(I-V)$ characteristics were measured at room temperature in the dark (Fig. 3), showing a striking dependence on the length of the metal gap $L$. For wide gaps, $(L=800 \mathrm{~nm})$, we find a symmetric non-ohmic behavior and a clear hysteresis loop in the downward-upward sweeps. This behavior is found to be quite reproducible for wide contact gaps $(800 \mathrm{~nm}$ at the bottom of Fig. 3). We found that the disordered guanosine films always behave like a rectifying barrier and induce a capacitor effect in the planar device, independent of the actual morphology of the film and device size.

The situation changes dramatically in the $120 \mathrm{~nm}$ device. In this case just a few SAGCs are probed by the tips. The $I-V$ characteristics show a non-linear symmetric behavior with a zero current region in the voltage range between -2 and $+2 \mathrm{~V}$. For bias higher than $2 \mathrm{~V}$ the current increases to a sub- $\mu \mathrm{A}$ level with a dynamical resistance of the order of a fraction of $\mathrm{M} \Omega$, 
(a)

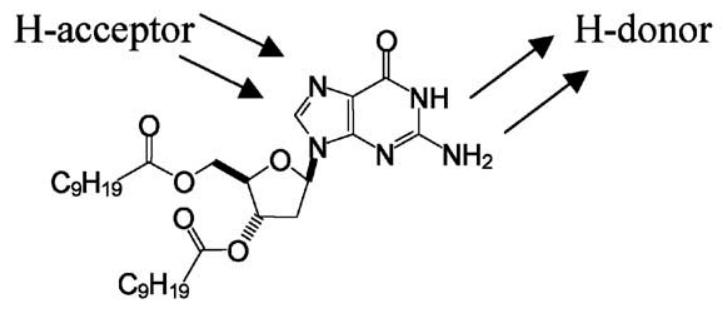

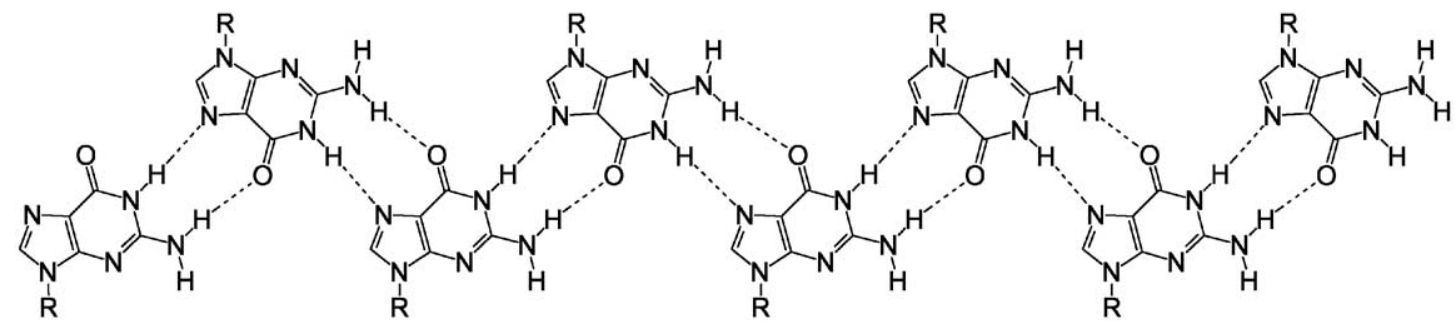

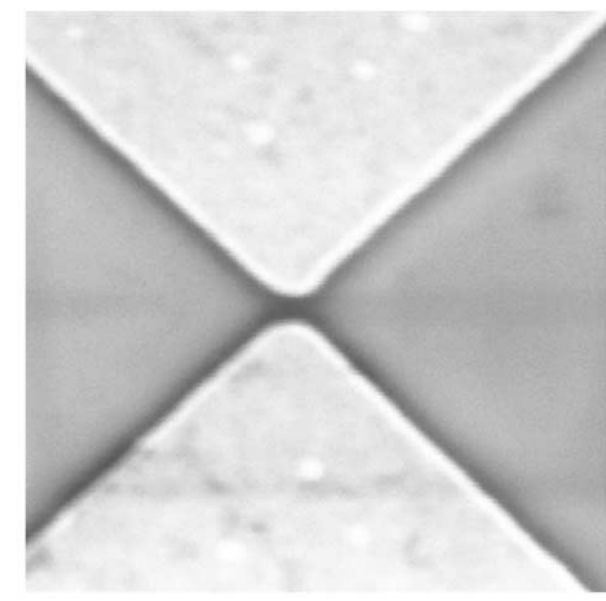

(b)

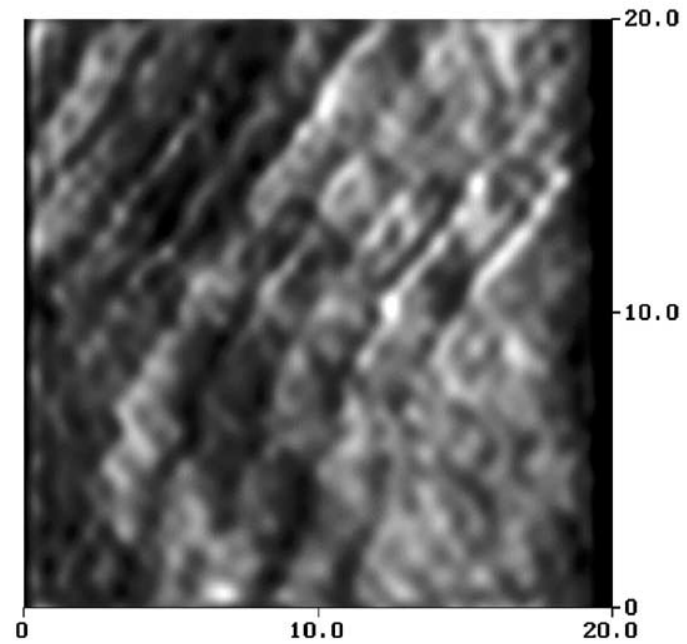

(c)

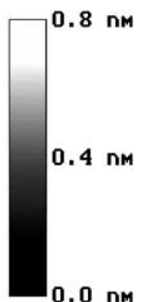

$\mathrm{nm}$

Fig. 1. (a) Individual deoxiguanosine molecule (top) and a typical ribbon-like aggregate of the molecule (bottom); (b) top-view SEM image of the gold nanoelectrodes (the spacing is $60 \mathrm{~nm}$ ); and (c) AFM image of the molecular layer deposited in the gap between the two electrodes. The AFM measurements were performed in contact mode in air.

and then it saturates. The shape of the $I-V$ in this case is similar to that of the metal-semiconductor-metal (MSM) device, and exhibits a very high photoresponsivity of about $1 \mathrm{~W} / \mathrm{A}$. Note that in this case the hysteresis is considerably reduced, as expected from the reduction of the capacity due to the few SAGCs probed in the gap.

A further change of the transport characteristics occurs for contact gaps of $60 \mathrm{~nm}$ (or less), where only one SAGC is probed. Under this condition the device exhibits a clear diode-like characteristic, with currents of the order of few $\mu \mathrm{A}$ for positive biases (at $10 \mathrm{~V}$ ) and $\mathrm{nA}$ for negative biases, without hysteresis. The onset of the diode occurs around $0.8 \mathrm{~V}$, with ideality factor $\eta \approx 4$. The observation of an asymmetric $I-V$ characteristic in devices where a single nanocrystal is probed suggests that a strong dipole is formed in each SAGC. This originates from the strong dipole of the 


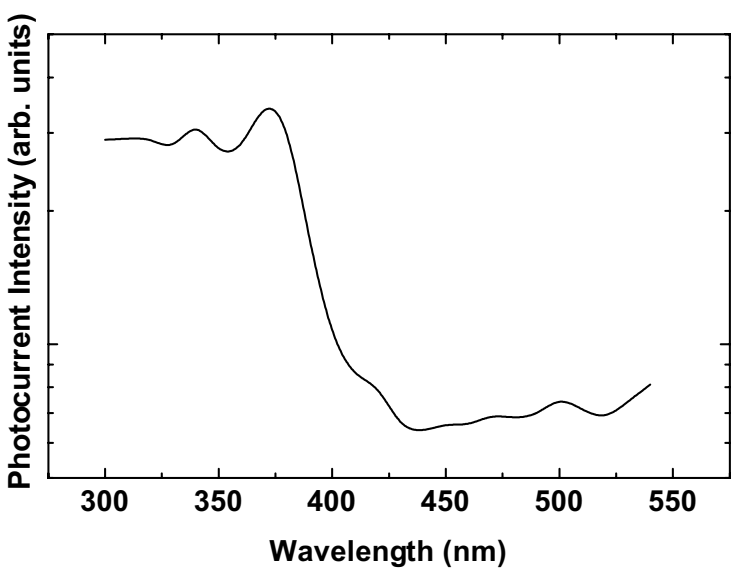

Fig. 2. Photocurrent spectrum of the SAGC measured with $-1 \mathrm{~V}$ bias.

guanosine molecules, oriented along the ribbon axis of the SAGCs. No transport is observed in open contacts without SAGCs, discarding the occurrence of arching or tunneling through the empty gap.

We should mention that the $I-V$ characteristics of Fig. 3 were reproducibly measured in several tens of devices. Moreover, we have not observed any aging of the SAGCs after several $I-V$ cycles in the range $\pm 6 \mathrm{~V}$ in air and at room temperature.

The strong differences in the characteristics of the devices depending on $L$, suggest that different conduction mechanisms concur to the overall charge transport depending on the relative variation of the gap width versus the self-assembling length. Since the SAGCs spontaneously form on a scale length of about $100 \mathrm{~nm}$, the narrow contacts probe just a single SAGC, where band-like transport through the $\pi-\pi$ stack occurs. This results in a temperature-dependent conductivity similar to that of semiconductors (see Fig. 4a)

$\sigma_{\mathrm{DC}}=\frac{n e^{2} \tau}{m}=\sigma_{0} \mathrm{e}^{-E_{G} / 2 k T}$

assuming a constant mobility.

The wider contacts probe several SAGCs randomly distributed in the gap, so that hopping among adjacent nanocrystals must occur to have transport. In this case temperature-dependent conductivity is similar to that of polycrystalline and polymer materials (see Fig. 4b)

$\sigma_{\mathrm{DC}}=\sigma_{0} \mathrm{e}^{-\left(T_{0} / T\right)^{\gamma}}$,

where the values of $\gamma$ and $T_{0}$ depend on the specific hopping process and on the hopping barrier
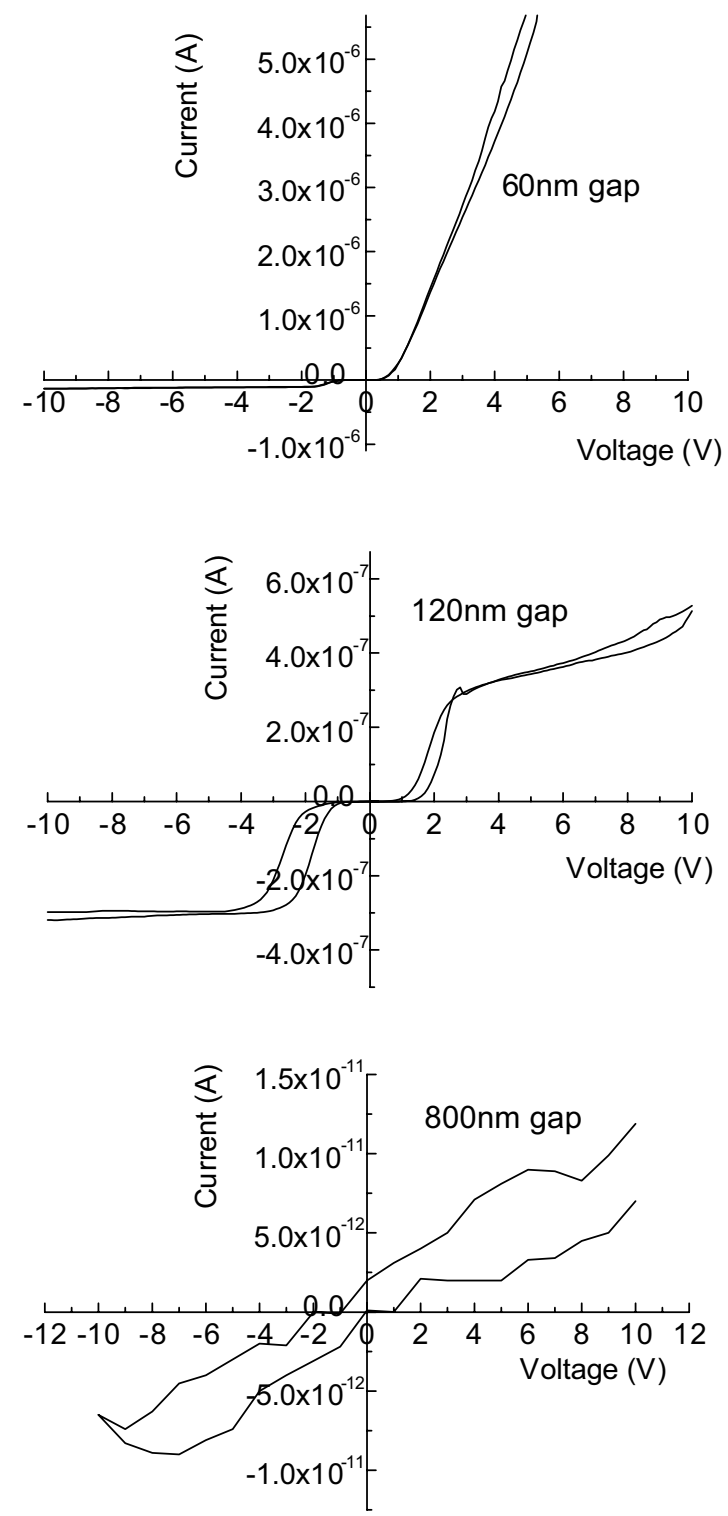

Fig. 3. Current-voltage characteristics measured in three devices with gaps of 60,120 and $800 \mathrm{~nm}$ at room temperature and in air.

( $T_{0}$ amounts to several hundreds of degree Kelvin). In particular, the $\gamma$ value equal to 0.45 is very close to the theoretical value predicted by the Efros-Shklovskii theory for amorphous and disordered materials (see e.g. Ref. [13]).

Noticeably, the photosensitivity of the $120 \mathrm{~nm}$ device is independent of the light wavelength, supporting the idea of a photon assisted incoherent 

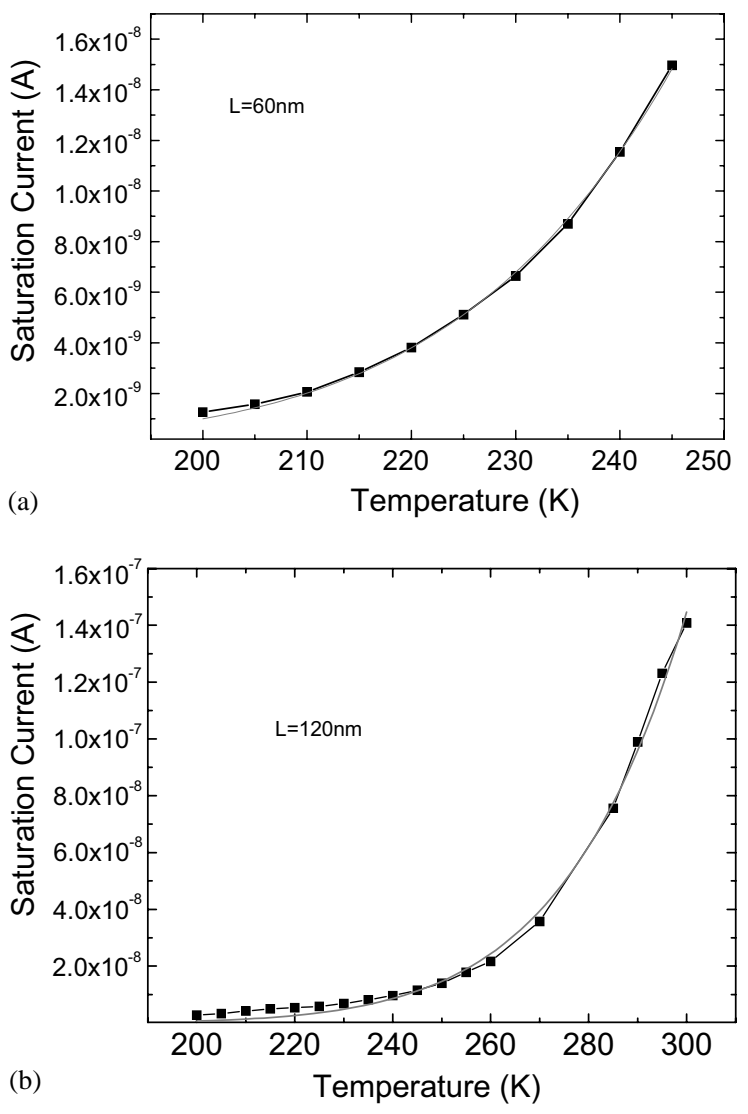

Fig. 4. Conductivity of a $60 \mathrm{~nm}$ contact (a) and of a $120 \mathrm{~nm}$ contact (b). The curves through the datapoints are the results of Eqs. (1) and (2), respectively.

hopping governing the transport. The control of the self-organization on a scale length of the order of $250 \mathrm{~nm}$, would enable the fabrication of such devices by simple ink-jet printing of the guanosine solution on a pattern realized by optical lithography.

\section{The bottom-up approach}

In the bottom-up approach we use a metalloprotein such as azurin. The main feature of this protein is the presence of a $\mathrm{Cu}$ atom in the redox active site which endows the molecule with its electron transfer properties (the big-dot at the center of the molecule in Fig. 5). Such blue copper proteins appear to be suitable candidates for biomolecular nanoelectronics applications due to their natural electron transfer ac-

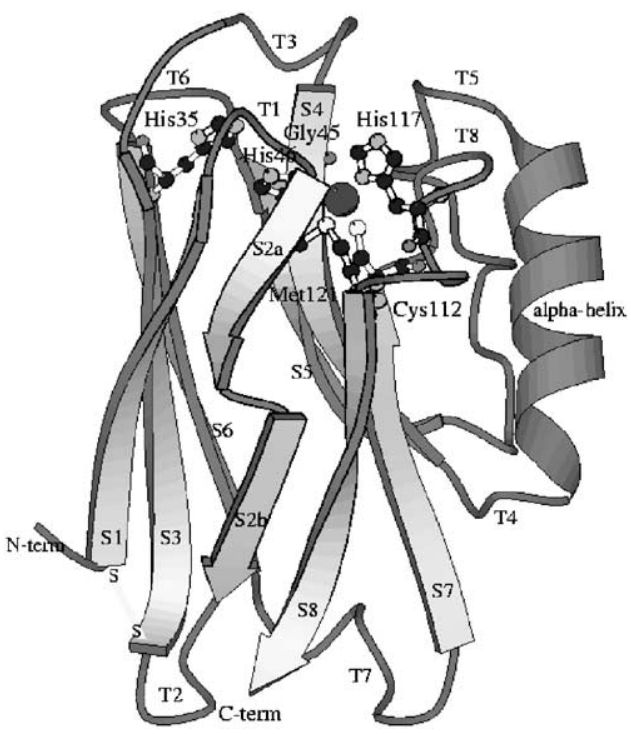

Fig. 5. Scheme of the azurin protein. The big blue dot at the center is the copper atom.

tivity in biological environments. This typical functionality connected with their redox nature makes the metalloproteins, and particularly blue copper proteins such as azurin, plastocyanin, and their suitably designed mutants, promising candidates for the realization of molecular switches whose conduction state can be controlled by tuning their redox state with the help of an external voltage source (gate). The protein has two cysteine groups and 12 amino groups which can be used to bind the protein to properly functionalized surfaces, thus allowing us to deposit oriented layers on semiconductors, oxides and metals, opening up the way to electronic applications. In order to realize a biomolecular device, a very precise study of the redox, electronic and electrical properties of these metalloprotein linked to an inorganic substrate under different, non-physiological environments is required.

To this aim we deposited azurin layers on $\mathrm{SiO}_{2}$ substrates between two gold electrodes separated by $50 \mathrm{~nm}$, similar to the planar structure adopted for the guanosine (Fig. 1). In order to study the effect of the molecular ordering on the device transport we have developed two different immobilization processes. The first method acts on 12 possible amino-groups of the molecule (the ribbons labeled by $T_{i}$ in Fig. 5), resulting in a random immobilization of the proteins on the substrate. It is based on a 3-step 
reaction, first by incubating the $\mathrm{Si} / \mathrm{SiO}_{2}$ substrate in 3-aminopropyltriethoexysilane (3-APTS) for $2 \mathrm{~min}$ and then by rinsing in $\mathrm{CHCl}_{3}$ in order to remove 3-APTS molecules not linked to the surface. The second step consisted in exposing the sample already reacted with silanes to glutaralde (GD) for $10 \mathrm{~min}$ followed by a thorough washing in ultra-pure $\mathrm{H}_{2} \mathrm{O}$; finally-the pre-coated substrates were exposed to azurin solution for $20 \mathrm{~min}$ and rinsed in $\mathrm{NH}_{4} \mathrm{Ac}$ to get rid of physisorbed molecules.

Oriented immobilization was instead obtained on $\mathrm{Si} / \mathrm{SiO}_{2}$ substrates by adopting another process based on incubation with 3-mercaptopropyltrimethoxysilane (3-MPTS) for $2 \mathrm{~min}$, rinsed in $\mathrm{CHCl}_{3}$, then exposed to the azurin solution for typically 40-60 min, and thoroughly rinsed in pure buffer. Protein immobilization took place via the reaction of the free thiols groups of 3-MPTS with the surface disulphide group of azurin, giving rise to intermolecular disulfide bonds. Since only one disulfide bond is available in azurin for this reaction, the orientation of the molecule is expected to be well defined.

The current-voltage curves measured on samples with the ordered and disordered layer of azurin are shown in Figs. 6a and b, respectively. In both cases we observe a clear diode-behavior, with dynamic resistance of the order of 3-4 M $\Omega$. The rather large current values clearly indicate that the electron transfer mechanisms in the protein, between the $\mathrm{Cu}$ metallic site and the edges is quite effective in determining the conduction processes. More importantly, we see that the device with the ordered protein layer (Fig. 6a), exhibits current values about ten times larger than the device with disordered layer (Fig. 6b), and negligible hysteresis. The current-voltage hysteresis in the disordered devices is similar to that observed in the wide-gap guanosine devices (Fig. 3) and in the amorphous materials. Measurements performed after 1 day and 1 week of delay gave lower current intensities and changes in the value of the zero current region, indicating the extreme sensitivity of the metalloprotein to humidity and temperature variations.

\section{Conclusions}

We have briefly overviewed how to fabricate electronic devices by interconnecting self-organized
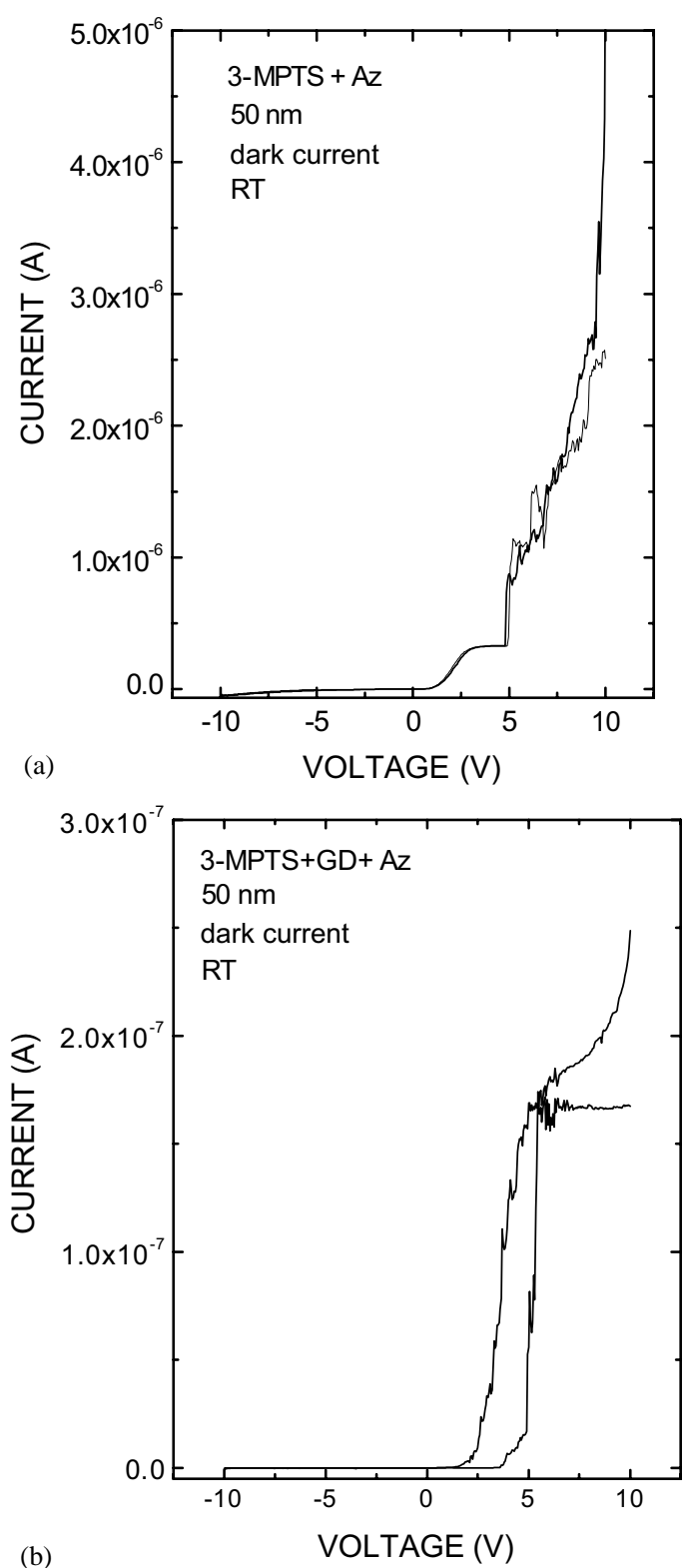

Fig. 6. $I-V$ curves (downward and upward sweeps) of the azurin devices with ordered (a) and disordered (b) layers. The disordered layer (b) exhibits strong hysteresis. The upward voltage sweep is the right curve, the downward voltage sweep is the left curve.

biomolecules and metallic nanopatterns. Both the macroscopic connection of self-ordered films and the chemical immobilization of metalloproteins can be used to realize basic devices operating at room 
temperature. The next step of this research will be the fabrication of a field effect transition, presently underway in our laboratory.

\section{Acknowledgements}

We gratefully acknowledge the collaboration with E. Molinari and R. DeFelice at INFM Modena Italy, G. Gottarelli and G.P. Spada at Bologna University Italy, P. Facci at INFM Viterbo Italy, and G. Canters and M. Verbeet at Leiden University (Holland). This research is granted by INFM through PRA "Sinprot" and by EC through the V-frame FET project "SAMBA".

\section{References}

[1] W. Henk, et al., Science 293 (2001) 76.

[2] R.M. Metzger, et al., Angew. Chem. Int. Ed. Engl. 40 (2001) 1749.

[3] C. Joachin, et al., Nature 408 (2000) 541.

[4] J. Chen, et al., Appl. Phys. Lett. 77 (2000) 1224.

[5] E. Braun, et al., Nature 391 (1998) 775.

[6] Y. Okahata, et al., J. Am. Chem. Soc. 120 (1998) 6165.

[7] D. Porath, et al., Nature 403 (2000) 635.

[8] R. Cingolani, Nature (Biotechnology) 18 (2000) 829.

[9] S.R. Whaley, et al., Nature 405 (2000) 665.

[10] S. Brown, et al., J. Mol. Biol. 2001, in press.

[11] R. Rinaldi, et al., Appl. Phys. Lett. 78 (2001), in press.

[12] See Craighead group web page www.hgc.cornell.edu

[13] J.A. Reedijk, H.C.F. Martens, H.B. Brons, M.A.J. Michels, http://arXiv.org (cond. Mat/9909398). 